\title{
ROBUST OPTICAL DESIGN OF ANGLED MULTILAYER DIELECTRIC MIRRORS OPTIMIZED FOR RUBIDIUM VAPOR CELL RETURN REFLECTION
}

\author{
M.A. Perez ${ }^{1}$, J. Kitching ${ }^{2}$ and A.M. Shkel ${ }^{1}$ \\ ${ }^{1}$ MicroSystems Labratory, University of California, Irvine, CA, USA \\ ${ }^{2}$ Time and Frequency Division, National Institute of Standards and Technology, Boulder, CO, USA
}

\begin{abstract}
This paper reports on the design and implementation of thin film multilayer dielectric to form Bragg reflectors on the sidewalls of micromachined atomic cells. Due to deposition shadowing, significant variations in the thicknesses of the thin films are encountered when the layers are deposited using PECVD. These gradients in thickness may limit optical performance of the reflector in atomic cells. An optimized design procedure is described to maximize the performance of the reflector at a wavelength of $795 \mathrm{~nm}$ under the variations in material thickness. In addition, an optical design based on two shifted quarter wave Bragg reflectors in series is used to form a reflector with extended optical bandwidth for added robustness. The extended reflectance range maintains high reflection at the $D_{1}$ absorption wavelength of ${ }^{87} R b$ despite variations in uniformity greater than $70 \%$. The developed reflector technology is ideally suited for use in miniature rubidium vapor cells. We demonstrate less than $1.5 \mathrm{~dB}$ of return loss with circular polarization ellipticity maintained to $\pm 2^{\circ}$ is demonstrated, as required for many atomic MEMS applications.
\end{abstract}

\section{INTRODUCTION}

Miniature vapor cells for emerging atomic MEMS applications, such as chip scale atomic clocks [1], magnetometers [2], [3] and gyroscopes, depend on the efficient routing of laser light using micromachined reflectors. Cells containing rubidium alkali vapor need low reflection losses at the ${ }^{87} R b D_{1}$ transition wavelength of $795 \mathrm{~nm}$ after multiple reflections inside the vapor cell cavity formed in bulk micromachined wet-etched silicon (Figure 1).

However, uncoated silicon is not sufficiently reflective for use as a high performance mirror, losing $67 \%$ of optical energy in bulk transmission. Previously, rubidium vapor cells with optical return performance superior to uncoated silicon have been demonstrated by use of multilayer dielectric reflectors integrated on the sidewalls of wet-etched silicon cavities fabricated using Plasma Enhanced Chemical Vapor Deposition (PECVD) [4]. Although these reflectors have the potential to reflect light with negligible loss, large variations in the thin film thicknesses were observed due to the deposition technology, which limited the reflector performance.

PECVD has many practical advantages over other thin film fabrication methods, such as Physical Vapor Deposition (PVD), including higher deposition rates and the formation of films with better mechanically and environmental robustness [5]. However,

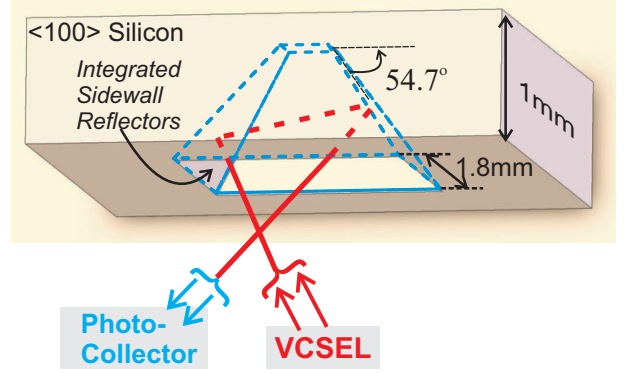

Fig. 1. Bulk micromachined reflector cell for atomic MEMS

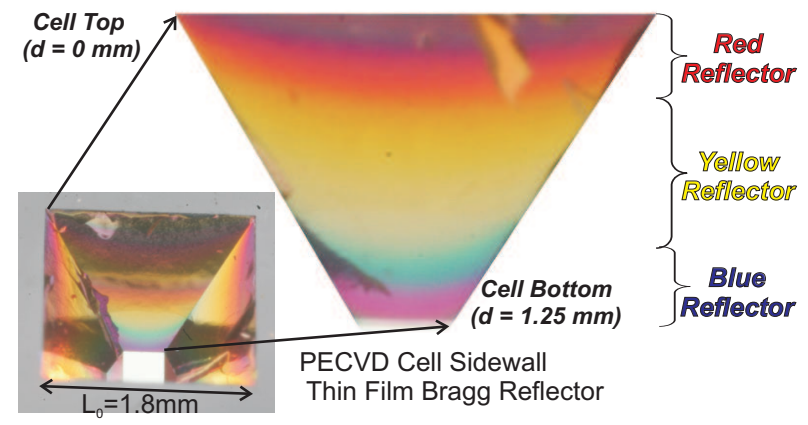

Fig. 2. PECVD Bragg reflector on the sidewall of a reflector cell with shifting wavelength reflectance from deposition nonuniformity

the thicknesses of PECVD fabricated thin films deposited onto the sidewalls of micromachined cavities rapidly decreases with cavity depth. This film thinning is due to the decrease in the arrival angle available to reactant species (shadowing) from the PECVD reactor chamber [6]. For the proposed vapor cell geometry, the resulting nonuniformity results in a reduction in layer thickness by over 70 $\%$ down the cavity's sidewall.

When a multilayer reflector is deposited on the angled sidewalls (in this case at $54.7^{\circ}$ ), this nonuniformity results in a shift in the reflection to lower wavelengths, as observed in the shift in the reflector color from red to yellow to blue down the sidewall face (Figure 2). Since the optimum reflectance wavelength varies over the area of the reflector, the efficiency at the design wavelength is compromised. By increasing the range of the high reflectance band (the range of wavelengths for which the reflectivity is optimized) and optimizing the deposition process for a particular wavelength, maximum reflectance is achieved down the entire sidewall at the design wavelength.

\section{REFLECTOR DESIGN}

\section{Bragg Reflectors}

The design of micromachined cells with integrated Bragg reflectors for rubidium vapor cells has been previously described in [4]. Briefly, the light reflected at the interface between each layer may be made to constructively interfere to maximize the total reflected optical power at a specific wavelength. The reflectivity is maximized if the structure is composed of alternating thin film layers each of optical thickness of one-quarter wavelength of the light to be reflected as given by [7]

$$
t=\frac{\lambda_{0}}{4 n},
$$

where $t$ is the thickness of each layer, $\lambda_{0}$ is the wavelength of light to be reflected and $n$ is the index of refraction of each layer.

The high reflectance wavelength bandwidth is given by [7]

$$
2 \Delta g=\frac{4}{\pi} \sin ^{-1}\left(\frac{n_{H} / n_{L}-1}{n_{H} / n_{L}+1}\right),
$$

where $g$ is the normalized wavenumber $\left(g=\lambda_{0} / \lambda\right)$ and $n_{H}$ is the higher and $n_{L}$ the lower index of refractions of each of the thin 


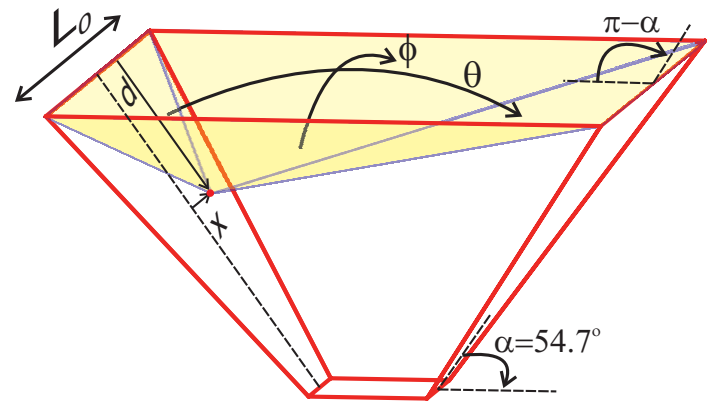

Fig. 3. Arrival angles on the sidewall of a reflector cell cavity

film materials. The wavelengths at the upper $\left(\lambda_{+}\right)$and lower $\left(\lambda_{-}\right)$ limits of this reflectance band are given by

$$
\begin{aligned}
& \lambda_{+}=\frac{\lambda_{0}}{1-\Delta g} \\
& \lambda_{-}=\frac{\lambda_{0}}{1+\Delta g},
\end{aligned}
$$

which yields the reflector bandwidth $\Delta \lambda$ in wavelength units by

$$
\Delta \lambda=\lambda_{+}-\lambda_{-}=\frac{2 \Delta g}{1-(\Delta g)^{2}} \lambda_{0} .
$$

The width of the reflectance band is maximized using high contrast ratio $\left(n_{H} / n_{L}\right)$ materials. Amorphous silicon $\left(\alpha S i, n_{H}=3.9\right)$ and silicon dioxide $\left(\mathrm{SiO}_{2}, n_{L}=1.45\right)$ are readily deposited using PECVD and yield a high optical index contrast $\left(\frac{n_{H}}{n_{L}}=2.7\right)$ and $\Delta g=0.3$, such that for $\lambda_{0}=795 \mathrm{~nm}, \Delta \lambda=520 \mathrm{~nm}$ [8].

\section{Cavity Shadowing in PECVD}

Commonly, PECVD process tools are designed and developed for use with planar substrates. Although PECVD is frequently used for conformal coverage over abrupt features such as the sidewalls of micromachined cavities, it has been shown that PECVD films deposited onto the sidewalls of etched cavities are not uniform and decrease with the depth into the trench [6]. These variations are attributed to the high mean free path of gas species during PECVD and limited film precursor migration along the sidewall surface.

The thin film deposition rate into a cavity is characterized by the arrival angles opposite $(\theta)$ and parallel $(\phi)$ to the deposition face open to the PECVD reactor vessel (Figure 3 ). The arrival angle $\theta$ is calculated by

$$
\theta=\sin ^{-1}\left\{\sqrt{\frac{L_{0}^{2} \sin ^{2}(\alpha)}{L_{0}^{2}+d^{2}-2 L_{0} d \cos (\alpha)}}\right\},
$$

where $d$ is the depth along the sidewall face, $L_{0}$ is the cavity opening width, $\alpha$ is the etch angle, and the oblique or acute angle solution for $\sin ^{-1}$ must be chosen to provide the physically suitable and continuous solution as a function of sidewall depth. Arrival angle $\phi$ is calculated by

$$
\phi=\tan ^{-1}\left\{\frac{L_{0} / 2+x}{d}\right\}+\tan ^{-1}\left\{\frac{L_{0} / 2-x}{d}\right\},
$$

where $x$ is the horizontal displacement from the sidewall centerline.

The product of the two arrival angles may be used to estimate the total effect of arrival angle shadowing. When normalized by the arrival angles available to a planar substrate $\left(\theta_{\perp}=\pi, \phi_{\perp}=\pi\right)$, the fractional change in the arrival angle may be used to estimate the change in the deposition rate onto a micromachined cavity sidewall from that onto a planar surface. This yields the ratio of thickness of a thin film on a cavity sidewall $t$ to the thickness on a planar surface $t^{\perp}$ according to

$$
\frac{t}{t^{\perp}}=\frac{\phi \theta}{\phi_{\perp} \theta_{\perp}}=\frac{\phi \theta}{\pi^{2}} .
$$

From (1), the thickness variation in (8) results in two optical characteristics for a cavity sidewall integrated reflector: The first is the discrete shift in the reflectance band between a reflector deposited on a planar surface and onto cavity sidewall. The second characteristic is the continuous shift of the reflectance band to lower wavelengths down the reflector face into the cavity.

\section{Optimized Bragg Reflector for Cavity Sidewall Integration}

PECVD process parameters are generally defined in terms of the planar deposition rate. Since the physical thickness of each layer will shift across the interface from planar to sidewall deposition, it is of interest to define the design wavelength for planar deposition $\lambda_{0}^{\perp}$ that will achieve a sidewall reflector design at wavelength $\lambda$. In addition, it is desirable to optimize the planar design to maximize the reflectance at $\lambda$ over as much of the sidewall as possible under the shift to lower wavelengths down the sidewall into the cavity.

The reflector may be optimized at $\lambda$ if it is equal to the wavelength at the lower limit of the reflectance band $\lambda_{-}$at the top of the cavity sidewall. This provides that the entire reflectance band is available for decreasing film thickness and shifts to lower reflectance band wavelengths. Using (1), (4), and (8) yields

$$
\frac{\phi \theta}{\phi_{\perp} \theta_{\perp}}=\frac{\lambda_{-}(1+\Delta g)}{\lambda_{0}^{\perp}} .
$$

Optimizing (9) by setting $\lambda=\lambda_{-}$and evaluating the left hand side for the shadowing at the top of the sidewall for $d=0$ yields

$$
\lambda_{0}^{\perp}=\frac{\lambda(1+\Delta g)}{1-\alpha / \pi},
$$

where $\alpha$ is evaluated in radian units. Equation (10) is used to determine the planar deposition design wavelength $\lambda_{0}^{\perp}$ which yields a reflector designed to maximize the reflectance at a wavelength $\lambda$ deposited on a sidewall at etch angle $\alpha$. For a $\alpha \mathrm{Si} / \mathrm{SiO}_{2}$ system with $\alpha=54.7^{\circ}$ and $\lambda=795 \mathrm{~nm}$, we find $\lambda_{0}^{\perp}=1480 \mathrm{~nm} . \lambda_{0}^{\perp}$ is used to establish the deposition time using the thin film thickness from (1) for the deposition rates in any particular PECVD system.

\section{Robustness to Film Thinning}

The reflection bandwidth may be used as a measure of the robustness to film thinning due to cavity shadowing when used in narrowband (laser) applications at a wavelength $\lambda$. Thinning thin film layer thicknesses will shift the reflection wavelength according to (1). If the variation in thickness causes a shift in the target wavelength $\lambda_{0}$ greater than the bandwidth of the reflector $\Delta \lambda$, the reflectance will be reduced at the target wavelength. Using (3) and (4) with (1) reflector wavelength limits over the fractional change in thin film thickness are evaluated according to

$$
\frac{t}{t_{0}}=\frac{(1-\Delta g)}{(1+\Delta g)}
$$

where $t_{0}$ is the thickness at $d=0$ and $\lambda \equiv \lambda_{-} \equiv \lambda_{+}$for a reflector ideally optimized to maximize reflectance at $\lambda$. For an $\alpha \mathrm{Si}_{/} \mathrm{SiO}_{2}$ Bragg reflector $t / t_{0}=0.54$, which indicates that the reflector may be optimized using (10) to be robust for up to a reduction in the thin film thicknesses of $46 \%$. 


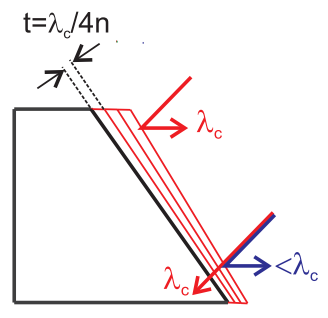

(a) Conventional

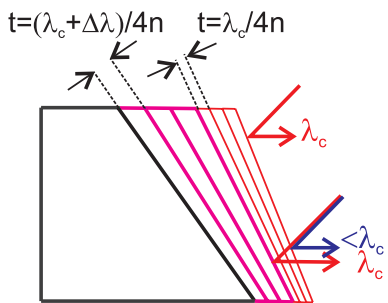

(b) Extended
Fig. 4. Bragg reflector design for deposition shadowing robustness

\section{Extended Bragg Reflector}

The variation in film thickness due to to cavity shadowing in a particular cavity geometry may be too large to maintain high reflectance at the target wavelength using a conventional Bragg reflector despite optimization. The effect of cavity shadowing on deposition rate variation is shown in Figure 5(a) for the geometry explored in this work, which produces a pattern nearly identical to the color variation observed by the naked eye on the sidewall of the reflector cell (Figure 2). At the bottom of the sidewall, the film thickness will decrease to less than $t / t_{0}=0.3$, resulting in a reduction in the thickness of the reflector films of more than 70 $\%$.

The effect of this shift in deposition rate on an optimized conventional Bragg reflector is shown in Figure 5(b). As the reduction in deposition rate exceeds $46 \%$, the reflectance band is shifted below $\lambda=795 \mathrm{~nm}$ and the reflectance is reduced to below $50 \%$ to as low as $10 \%$ on the lower face of sidewall reflector, becoming effectively transparent to the design wavelength over the lower region of the reflector (Figure 4(a)).

However, the bandwidth of the reflector may be extended by using two Bragg reflectors in series. A second, thicker Bragg reflector provides high reflectance at higher wavelengths and adds to the total reflectance bandwidth. The second reflectors then provides high reflectance in the region where the upper Bragg reflector is transparent (Figure 4(b)).

The design wavelength of the second reflector is optimally designed by shifting the design wavelength by the reflectance bandwidth to $\lambda_{0}^{S}$. Using (3) and (4) yields

$$
\frac{\lambda_{0}^{S}}{\lambda_{0}}=\frac{(1+\Delta g)}{(1-\Delta g)},
$$

where the upper reflectance band limit of the original reflector is matched to the lower limit of the second reflector $\left(\lambda_{+} \equiv \lambda_{-}^{S}\right)$ to

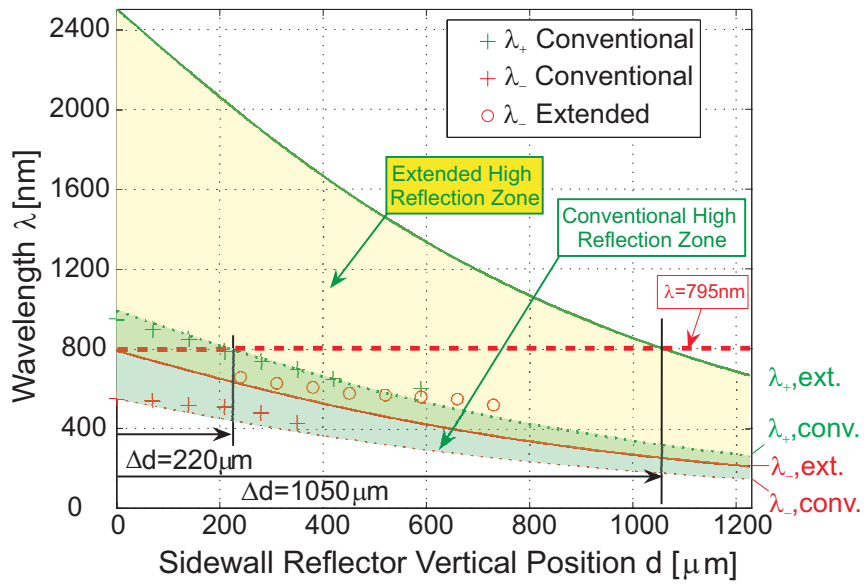

Fig. 6. Conventional vs. optimally designed Extended Bragg reflectors for use at $795 \mathrm{~nm}$ in micromachined reflector cell

create a composite reflector with an ideally extended reflectance to $2 \Delta g$. For the $\alpha \mathrm{Si} / \mathrm{SiO}_{2} \mathrm{Bragg}$ reflector, the shifted reflector is optimally designed with $\lambda_{0}^{S}=1.8 \lambda_{0}$, increasing the thickness of the layers by $80 \%$. From (11) and $2 \Delta g=0.6$, the extended reflector is able to maintain high reflectance over thickness reductions due to cavity shadowing down to $t / t_{0}=0.3$. As shown in Figure $5(\mathrm{c})$, the use of an extended band reflector allows $90 \%$ reflectance to be maintained down the sidewall for up to $70 \%$ thinning due to cavity shadowing.

\section{EXPERIMENTAL RESULTS}

Reflector cells were fabricated according to the process described previously in [4]. Briefly, a $1 \mathrm{~mm}$ thick $<100\rangle$ silicon wafer is wet through-etched in potassium hydroxide $(\mathrm{KOH})$ with a silicon nitride hardmask with $1.8 \mathrm{~mm}$ square lithographically patterned openings. This produces cavities with $54.7^{\circ}$ sidewalls formed by the $<111>$ crystallographic planes. Optical thin films are sequentially deposited onto the sidewalls via PECVD in a PlasmaTherm 790 under continuous vacuum forming reflectors.

The reflector is composed of two six layer Bragg reflectors in series with a total of 12 alternating $\alpha \mathrm{Si}$ and $\mathrm{SiO}_{2}$ thin films. The top (thinner) six layers are of $\alpha \mathrm{Si}$ and $\mathrm{SiO}_{2}$ with $90 \mathrm{~nm}$ and 240 $\mathrm{nm}$ planar deposition thicknesses, respectively. Upon deposition onto the sidewall of the cavity, the thicknesses of the films are expected to reduce by $30 \%$ according to (9), yielding estimated sidewall thicknesses of $60 \mathrm{~nm}$ and $170 \mathrm{~nm}$, respectively. These layers provide high reflectance over the upper section of the cell. The bottom (thicker) six layers are of $\alpha \mathrm{Si}$ and $\mathrm{SiO}_{2}$ with planar

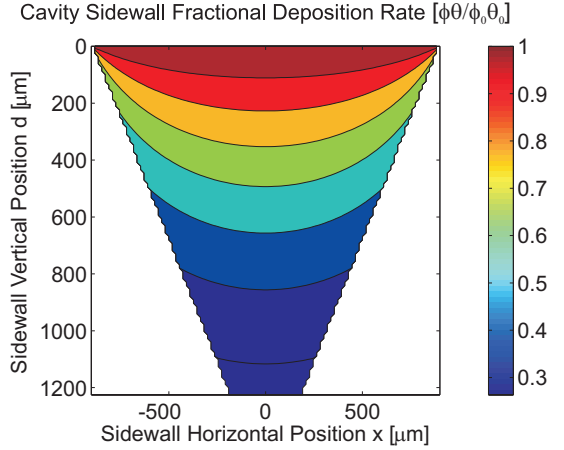

(a) Deposition rate variation

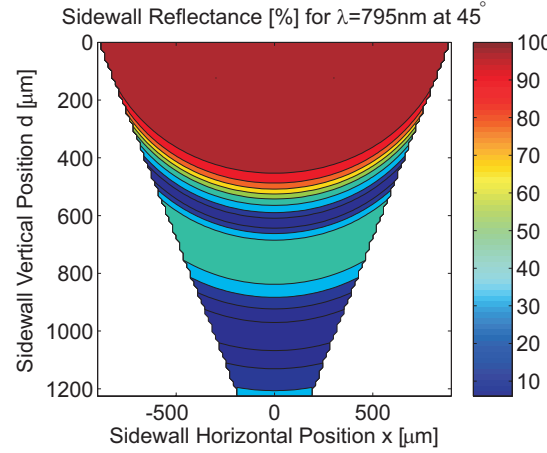

(b) Conventional: $3 \times\left[\alpha \mathrm{Si} \mathrm{SiO}_{2}\right]$ layers

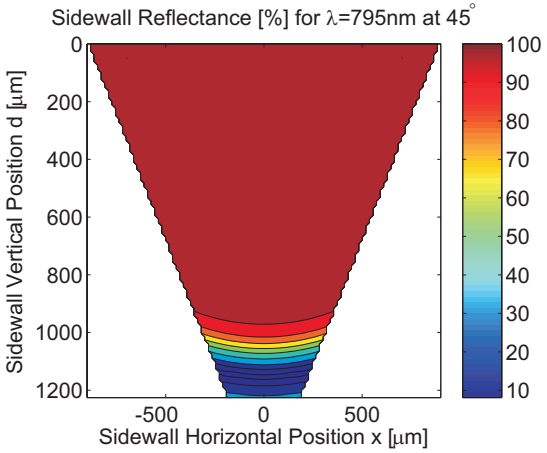

(c) Extended: $6 \times\left[\alpha \mathrm{Si} \mathrm{SiO}_{2}\right]$ layers

Fig. 5. Effect of cavity deposition shadowing on sidewall PECVD Bragg reflectors $\left(L_{0}=1.8 \mathrm{~mm}, \alpha=54.7^{\circ}\right)$ 
deposition thicknesses $170 \mathrm{~nm}$ and $430 \mathrm{~nm}$, sidewall thicknesses of $120 \mathrm{~nm}$ and $300 \mathrm{~nm}$, and provide high reflectance over the lower section of the cell.

The reflectance bandwidth of the cell reflector faces were characterized by using an Ocean Optics [9] USB2000 spectrometer with a reflection probe coupled to an LS-1 tungston halogen light source. The lower wavelength limits of the reflection bandwidth was extracted from this broadband characterization. This is compared to the wavelength limits for the previously fabricated six layer conventional reflector not optimized for sidewall deposition, as well as the analytically projected reflectance bandwidths, in Figure 6. The optimized reflector is demonstrated to maintain high reflectance at $795 \mathrm{~nm}$ over more than $85 \%$ of the cavity sidewall, compared to less than $20 \%$ for the previously fabricated conventional Bragg reflector.

The performance of a complete cell is demonstrated at $795 \mathrm{~nm}$ by mounting a sample on a rotation stage from which the circularly polarized collimated beam from a ULM [9] VCSEL is directed via the sidewall reflectors into a Thorlabs [9] PAX polarimeter, as shown in Figure 7. Return reflection demonstrates optical losses of less than $-1.5 \mathrm{~dB}$ and the polarization state is maintained to $\pm 2^{\circ}$ of ellipticity over incident angles from $10^{\circ}$ to $30^{\circ}$, as shown in Figure 8. This experimental results demonstrate applicability of the design to many atomic MEMS applications of interest, as both high reflectance and ellipticity maintenance is required for the propagation of circularly polarized light in those applications.

\section{CONCLUSIONS}

An optimized design procedure for the integration of multilayer PECVD reflectors onto the sidewalls of bulk micromachined cavities has been described for the fabrication of reflector cells with improved optical return performance. This procedure optimizes the reflectance bandwidth for the cavity geometry to maintain high reflectance under deposition non-uniformities caused by deposition shadowing. A design for an extended reflectance band was introduced by using two shifted Bragg reflectors in series. The design was shown to be effective in maintaining high reflection at the $D_{1}$ absorption wavelength of ${ }^{87} R b$ despite non-uniformities in excess of $70 \%$ for optical return applications. The fabricated cell was characterized to be able to return light at $795 \mathrm{~nm}$ with losses less than $1.5 \mathrm{~dB}$ while maintaining the polarization ellipticity of circular polarized light to $\pm 2^{\circ}$. In this paper we demonstrate an effective technique for the integration of multilayer reflectors with nonplanar microdevices, such as on the sidewalls of micromachined cavities. This work paves the way for the introduction of high performance optics into atomic MEMS sensors and other optical MEMS with nontrivial geometries.

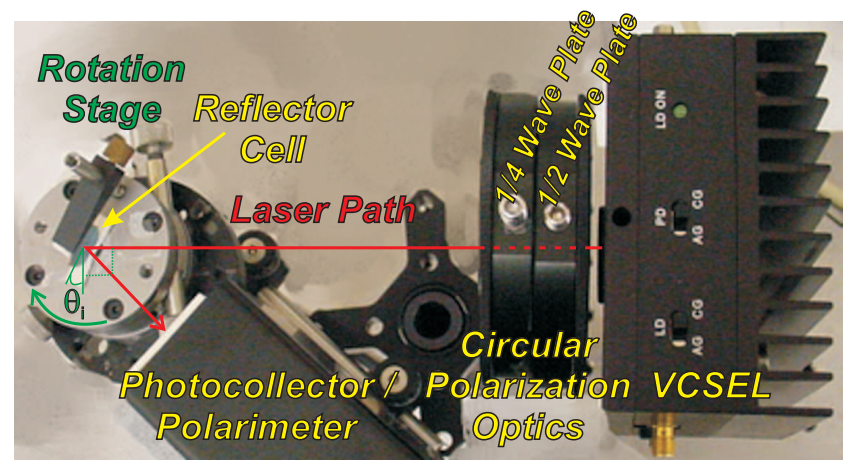

Fig. 7. Experimental stage for return reflection characterization of the Bragg reflector cell

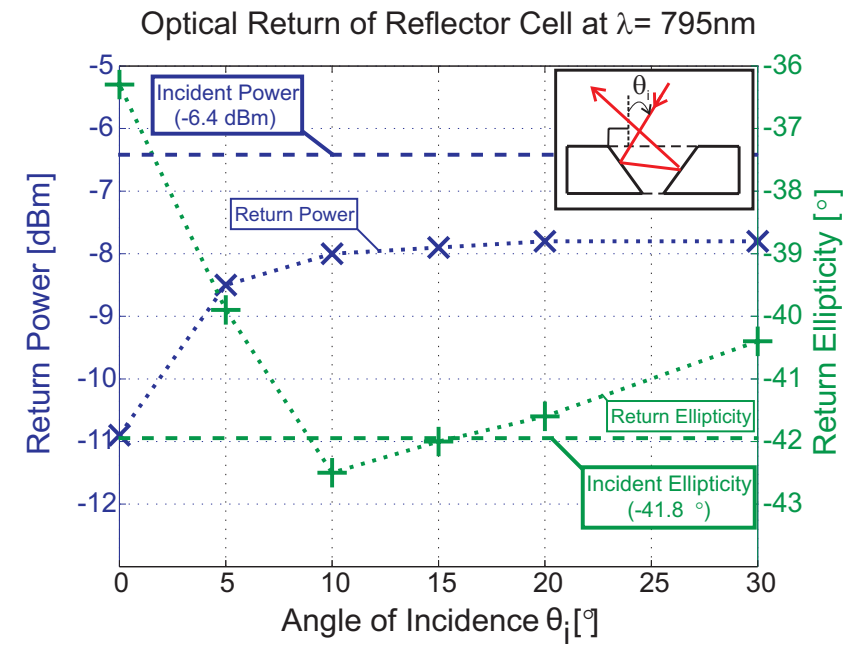

Fig. 8. Experimental return reflection characterization of the extended Bragg reflector cell

\section{ACKNOWLEDGMENT}

The authors thank the staff of the Integrated Nanosystems Research Facility (INRF) for fabrication assistance. This work was supported in part by the Defense Advanced Research Projects Agency (DARPA) Navigation-Grade Integrated Micro Gyroscopes (NGIMG) program and is a partial contribution of the National Institute of Standards and Technology (NIST), an agency of the US government, and is not subject to copyright.

\section{REFERENCES}

[1] D. Younger, L. Lust, D. Carlson, S. Lu, L. Forner, H. Chanhvongsak, and T. Stark, "A manufacturable chip-scale atomic clock," in Transducers '07 \& Eurosensors XXI: The 14th International Conference on Solid-State Sensors and Actuators and Microsystems, June 2007.

[2] L.-A. Liew, S. Knappe, J. Moreland, H. Robinson, L. Hollberg, and J. Kitching, "Microfabricated alkali atom vapor cells," Appl. Phys. Lett., vol. 84, p. 2694, 2004.

[3] S. Knappe, V. Gerginov, P. D. D. Schwindt, V. Shah, H. G. Robinson, L. Hollberg, and J. Kitching, "Atomic vapor cells for chip-scale atomic clocks with improved long-term frequency stability," Opt. Lett., vol. 30, pp. 351-2353, 2005.

[4] M. Perez, U. Nguyen, S. Knappe, E. Donley, J. Kitching, and A. Shkel, "Rubidium vapor cell with integrated nonmetallic multilayer reflectors," in IEEE 21st International Conference on Micro Electro Mechanical Systems, 2008. MEMS 2008, 13-17 January 2008, pp. 790-793.

[5] L. Martinu and D. Poitras, "Plasma deposition of optical films and coatings: A review," Journal of Vacuum Science \& Technology A: Vacuum, Surfaces, and Films, vol. 18, no. 6, pp. 2619-2645, 2000.

[6] A. Adams, "Plasma deposition of inorganic films," Solid State Technology, vol. 26, no. 4, pp. 135-139, April 1983.

[7] H. A. Macleod, Thin-Film Optical Filters, 3rd ed. Institute of Physics Publishing, Bristol-Philadelphia, 2001.

[8] E. D. Palik, Handbook of Optical Constants of Solids, E. D. Palik, Ed. Elsevier, 1998, vol. 1.

[9] "Products or companies named here are cited only in the interested of complete scientific description, and neither constitute nor imply endorsement by NIST or by the US government." 